\title{
Optical representation of thermal nuclear fluctuation effect on band-gap renormalization
}

\author{
Kohei Ishii $\odot, *$ Jun Haruyama $\odot$, and Osamu Sugino $\odot$ \\ Institute for Solid State Physics, The University of Tokyo, Kashiwa, Chiba 277-8581, Japan
}

(Received 5 August 2021; revised 10 December 2021; accepted 14 December 2021; published 29 December 2021)

\begin{abstract}
The bandgap of insulating materials is renormalized in various ways by the electron-phonon interaction owing to the dynamical and quantum fluctuations of nuclei. These fluctuation effects are considered in the perturbative Allen-Heine-Cardona theory using the formulas for the Fan-Migdal and Debye-Waller terms. However, the material dependence is not clear in the formulas. Thus, in this study, we focus on the analytical form of the Debye-Waller term and find that the term can be reformulated using the momentum matrix. In addition, the optical selection rule is found to play a role. For diamond-type materials, the Debye-Waller term can be approximately decomposed into a product of the optical transition energy, the mean square displacement of nuclei, and the dipole transition probability. The decomposition can also be applied with an additional approximation to zinc-blende-type materials, as revealed by our first-principles calculation. The magnitudes of the Debye-Waller term of several materials can thus be estimated using basic physical quantities prior to performing the calculation of the electron-phonon interaction.
\end{abstract}

DOI: 10.1103/PhysRevB.104.245144

\section{INTRODUCTION}

The electron-phonon interaction (EPI) plays an important role in many areas of condensed matter physics such as semiconductor physics and superconductivity [1]. Considerable EPI effects can be observed in the bandgaps of solids at zero temperature and in the temperature dependence of these bandgaps. For example, the effect, or the bandgap renormalization, on diamond amounts to a few hundred $\mathrm{meV}$, as measured spectroscopically in the temperature range of $100-700 \mathrm{~K}$. Further, it is $320-450 \mathrm{meV}$ even at zero temperature due to the zero-point motion of nuclei [2]. Therefore, the accurate prediction of the bandgap requires a precise calculation of the EPI in addition to the calculation of the optical transition energy without including the EPI. Consequently, first-principles prediction had started approximately a decade after the 1990s when the density functional perturbation theory of the EPI was established [3] even though basic electronic structure theory was already established in the 1980s. Thus, the electron-phonon renormalization (EPR) is a relatively new topic in the field of first-principles calculation.

Prior first-principles calculations have been categorized as perturbative, which are based on the Allen-Heine-Cardona (AHC) theory developed around 1980 [4-6], and nonperturbative, where nuclear configurations are displaced stochastically with a Monte Carlo sampling technique [7,8], determined as a single thermally distorted configuration $[9,10]$, or described with a path integral molecular dynamics simulation [11]. The AHC theory can be traced back to 1951 when Fan studied the temperature dependent EPR by including lattice vibrations perturbatively [12] and to 1955 when Antončík proposed to alternatively include the degree of thermal nuclear

*Corresponding author: kishii@issp.u-tokyo.ac.jp vibration as an effective parameter called the Debye-Waller factor [13]. These two methods were unified by Allen, Heine, and Cardona between 1976 and 1983 [4-6] within the rigidion approximation (RIA), wherein potential sensed by an electron is decomposed into the contributions from each nucleus. The Fan's and Antončík's terms can be derived as the same perturbation order terms, and they are presently called the Fan-Migdal (FM) and Debye-Waller (DW) terms, respectively [1]. Further, the AHC theory was first applied to diamond in 2010 [14] and subsequently to various semiconductors, such as GaN, $\mathrm{Si}, \mathrm{BN}, \mathrm{AlN}, \mathrm{P}, \mathrm{LiF}, \mathrm{MgO}, \mathrm{LiNbO}_{3}$, AlAs, AlP, AlSb, GaP, and SiC [15-20].

The off-diagonal terms of the electronic self-energy were neglected in these studies but were recently made accessible for the first-principles calculation by the work of Lihm and Park [21]. They enabled this by rewriting the DW term with the momentum operator considering the translational invariance of the one-electron potential, along with enabling the consideration of the hybridization of electronic states. Consequently, this method was implemented in opensource software for first-principles calculations: QUANTUM ESPRESSO [22,23].

In this paper, we further rewrite the formulation of Lihm and Park [21] to transparently relate the DW term with optical transitions. This is realized by decomposing the DW term into terms contributed by each orbital and subsequently rewriting the orbital-resolved Debye-Waller (ORDW) terms using the momentum matrix. Consequently, the optical selection rule (OSR) plays a role in characterizing the DW term, which allows us to describe the DW term of diamond-type materials as a sum of the products of the optical transition energy, the mean square displacement (MSD) of nuclei, and the dipole transition probability under a plausible approximation of the lattice vibrations. Moreover, the approximate decomposition can be applied to zinc-blende-type materials, as revealed by our first-principles calculation. Thus, it is possible to estimate 
the magnitude of the DW term using basic physical quantities prior to performing the calculation of the EPI.

The structure of this paper is as follows: In Sec. II, the AHC theory (II A), the ORDW term and OSR (II B), and computational details (IIC) are explained. In Sec. III, we present an approximate form of the DW term (III A), the numerical validation of the bandgap EPR, and the origin of the material dependence of the ORDW term (III B). In Sec. IV, we present the conclusion of this paper.

\section{THE EPR OF THE ELECTRONIC STRUCTURES}

In this paper, we primarily follow the notation of Ref. [1] for the Born-von-Kármán boundary condition, BornOppenheimer approximation, Kohn-Sham (KS) method, and the harmonic approximation to lattice vibrations, which are briefly described as follows: We denote the $\alpha$-th component of the position of a supercell as $T_{\alpha}$ or, in the vector notation, as $\mathbf{T}$. The position of a $p$-th unit cell $\left(p=1, \cdots, N_{p}\right)$ is denoted as $\mathbf{R}_{p}$ and the equilibrium position of the $\kappa$-th nucleus in the unit cell is denoted as $\boldsymbol{\tau}_{\kappa p}^{0}$, which can be decomposed as $\boldsymbol{\tau}_{\kappa p}^{0}=\mathbf{R}_{p}+\boldsymbol{\tau}_{\kappa}^{0}$. The displacement from $\boldsymbol{\tau}_{\kappa p}^{0}$ is denoted as $\Delta \hat{\boldsymbol{\tau}}_{\kappa p}$ when it is treated as the position operator. Further, we denote the $n$-th KS state as $\left|\psi_{n \mathbf{k}}\right\rangle$, where $\mathbf{k}$ is the Bloch wavevector in the first Brillouin zone (FBZ), and the periodic part of $\left|\psi_{n \mathbf{k}}\right\rangle$ as $\left|u_{n \mathbf{k}}\right\rangle$. The KS equation is expressed as

$$
\left[-\hat{\nabla}^{2}+V^{\mathrm{KS}}\left(\hat{\mathbf{r}} ;\left\{\boldsymbol{\tau}_{\kappa p}^{0}\right\}\right)\right]\left|\psi_{n \mathbf{k}}\right\rangle=\varepsilon_{n \mathbf{k}}\left|\psi_{n \mathbf{k}}\right\rangle,
$$

where $\varepsilon_{n \mathbf{k}}$ is the KS eigenvalue and $V^{\mathrm{KS}}\left(\hat{\mathbf{r}} ;\left\{\boldsymbol{\tau}_{\kappa p}^{0}\right\}\right)$ is the effective KS potential for the equilibrium nuclear configuration:

$$
\begin{aligned}
V^{\mathrm{KS}}\left(\mathbf{r} ;\left\{\boldsymbol{\tau}_{\kappa p}^{0}\right\}\right)= & \sum_{\kappa, p, \mathbf{T}} V_{\kappa}\left(\mathbf{r}-\boldsymbol{\tau}_{\kappa p}^{0}-\mathbf{T}\right) \\
& +\sum_{\mathbf{T}} \int_{\mathrm{sc}} \frac{d \mathbf{r}^{\prime} n\left(\mathbf{r}^{\prime} ;\left\{\boldsymbol{\tau}_{\kappa p}^{0}\right\}\right)}{\left|\mathbf{r}-\mathbf{r}^{\prime}-\mathbf{T}\right|} \\
& +\left.\frac{\delta E^{x c}[n]}{\delta n}\right|_{n\left(\mathbf{r} ;\left\{\boldsymbol{\tau}_{\kappa p}^{0}\right\}\right)},
\end{aligned}
$$

where $V_{\kappa}$ is the pseudopotential of the nucleus $\kappa$, sc means "supercell", $n\left(\mathbf{r} ;\left\{\boldsymbol{\tau}_{\kappa p}^{0}\right\}\right)$ is the electron density at the equilibrium configuration, and $E^{x c}[n]$ is the exchange-correlation energy of $n . V_{\kappa}$ is a nonlocal pseudopotential, but nonlocality is not important in this paper, so it is written as a function of $\mathbf{r}$ for simplicity of notation. The interatomic force constant is denoted as

$$
C_{\kappa \alpha p, \kappa^{\prime} \alpha^{\prime} p^{\prime}}=\left.\frac{\partial^{2} E_{\mathrm{tot}}\left(\left\{\boldsymbol{\tau}_{\kappa p}\right\}\right)}{\partial \tau_{\kappa \alpha p} \partial \tau_{\kappa^{\prime} \alpha^{\prime} p^{\prime}}}\right|_{\left\{\boldsymbol{\tau}_{\kappa p}^{0}\right\}},
$$

where $E_{\mathrm{tot}}$ is the total-energy, and the dynamical matrix is denoted as

$$
D_{\kappa \alpha, \kappa^{\prime} \alpha^{\prime}}(\mathbf{q})=\left(M_{\kappa} M_{\kappa^{\prime}}\right)^{-1 / 2} \sum_{p} C_{\kappa \alpha 0, \kappa^{\prime} \alpha^{\prime} p} \exp \left(i \mathbf{q} \cdot \mathbf{R}_{p}\right)
$$

where $M_{\kappa}$ is the mass of nucleus $\kappa$. Then, the eigenvalue equation of a phonon is written as

$$
\sum_{\kappa^{\prime}, \alpha^{\prime}} D_{\kappa \alpha, \kappa^{\prime} \alpha^{\prime}}(\mathbf{q}) e_{\kappa^{\prime} \alpha^{\prime}, v}(\mathbf{q})=\omega_{\mathbf{q} \nu}^{2} e_{\kappa \alpha, v}(\mathbf{q}),
$$

where $e_{\kappa \alpha, v}(\mathbf{q})$ and $\omega_{\mathbf{q} v}$ are the eigenvector and eigenvalue, respectively, of the phonon at a wave -vector $\mathbf{q}$ in FBZ and a branch $v$.

\section{A. The AHC theory}

In the AHC theory, the EPR of the one-electron energy $\varepsilon_{n \mathbf{k}}$ at temperature $T$ is described as a sum of the FM term and DW term by

$$
\Delta \varepsilon_{n \mathbf{k}}(T)=\Delta \varepsilon_{n \mathbf{k}}^{\mathrm{FM}}(T)+\Delta \varepsilon_{n \mathbf{k}}^{\mathrm{DW}}(T),
$$

as derived by neglecting the off-diagonal terms of the electron self-energy. The FM term corresponds to the dynamical correction to $\varepsilon_{n \mathbf{k}}$ and has a form

$$
\begin{aligned}
\Delta \varepsilon_{n \mathbf{k}}^{\mathrm{FM}}(T)= & \operatorname{Re} \frac{1}{N_{p}} \sum_{m} \sum_{\mathbf{q}, v} \sum_{\kappa, \alpha, \kappa^{\prime}, \alpha^{\prime}} l_{\mathbf{q} v}^{2} \frac{M_{p}}{\sqrt{M_{\kappa} M_{\kappa^{\prime}}}} e_{\kappa \alpha, v}^{*}(\mathbf{q}) e_{\kappa^{\prime} \alpha^{\prime}, v}(\mathbf{q})\left[h_{m n, \kappa \alpha}(\mathbf{k}, \mathbf{q})\right]^{*} h_{m n, \kappa^{\prime} \alpha^{\prime}}(\mathbf{k}, \mathbf{q}) \\
& \times\left[\frac{1-f_{m \mathbf{k}+\mathbf{q}}(T)+n_{\mathbf{q} v}(T)}{\varepsilon_{n \mathbf{k}}-\varepsilon_{m \mathbf{k}+\mathbf{q}}-\omega_{\mathbf{q} v}+i \eta}+\frac{f_{m \mathbf{k}+\mathbf{q}}(T)+n_{\mathbf{q} v}(T)}{\varepsilon_{n \mathbf{k}}-\varepsilon_{m \mathbf{k}+\mathbf{q}}+\omega_{\mathbf{q} v}+i \eta}\right] .
\end{aligned}
$$

Here, $l_{\mathbf{q} v}=\left(2 M_{p} \omega_{\mathbf{q} v}\right)^{-1 / 2}$ is the zero-point length, $M_{p}$ is the reference mass that is considered as the proton mass, and $\eta$ is the convergence factor. $f_{m \mathbf{k}+\mathbf{q}}(T)=\left[e^{\left(\varepsilon_{m \mathbf{k}+\mathbf{q}}-\varepsilon_{F}\right) / k_{B} T}+1\right]^{-1}$ and $n_{\mathbf{q} v}(T)=\left(e^{\omega_{\mathbf{q} v} / k_{B} T}-1\right)^{-1}$ are the occupation numbers of electrons and phonons, respectively. $h_{m n, \kappa \alpha}(\mathbf{k}, \mathbf{q})$ is defined as

$$
h_{m n, \kappa \alpha}(\mathbf{k}, \mathbf{q})=\left\langle u_{m \mathbf{k}+\mathbf{q}}\left|\partial_{\kappa \alpha, \mathbf{q}} \hat{V}^{\mathrm{KS}}\right| u_{n \mathbf{k}}\right\rangle_{\mathrm{uc}},
$$

where $\partial_{\kappa \alpha, \mathbf{q}} \hat{V}^{\mathrm{KS}}$ is defined by

$$
\partial_{\kappa \alpha, \mathbf{q}} V^{\mathrm{KS}}(\mathbf{r})=\left.\sum_{p} e^{-i \mathbf{q} \cdot\left(\mathbf{r}-\mathbf{R}_{p}\right)} \frac{\partial V^{\mathrm{KS}}\left(\mathbf{r} ;\left\{\boldsymbol{\tau}_{\kappa p}^{0}\right\}\right)}{\partial \tau_{\kappa \alpha}^{0}}\right|_{\mathbf{r}-\mathbf{R}_{p}},
$$

and uc indicates that the integration is performed over the unit cell. Further, the DW term originates from the static correction to the one-electron potential, which arises from the distribution of nuclei around their equilibrium positions. According to Ref. [21], it has the following form under the RIA [24]:

$$
\begin{aligned}
\Delta \varepsilon_{n \mathbf{k}}^{\mathrm{DW}}(T)= & \frac{i}{N_{p}} \sum_{\mathbf{q}, v} \sum_{\kappa, \alpha, \alpha^{\prime}}\left\langle u_{n \mathbf{k}}\right|\left[\partial_{\kappa \alpha, \mathbf{0}} \hat{V}^{\mathrm{KS}}, \hat{p}_{\alpha^{\prime}}\right] \\
& \times\left|u_{n \mathbf{k}}\right\rangle l_{\mathbf{q} v}^{2} \frac{M_{p}}{M_{\kappa}} e_{\kappa \alpha, v}^{*}(\mathbf{q}) e_{\kappa \alpha^{\prime}, v}(\mathbf{q})\left[n_{\mathbf{q} v}(T)+\frac{1}{2}\right],
\end{aligned}
$$


where $\hat{p}_{\alpha}=-i \partial / \partial \hat{r}_{\alpha}$ is the $\alpha$-th component of the momentum operator.

\section{B. The ORDW term and the OSR}

We then rewrite the DW term by inserting the closure relation $\sum_{m, \mathbf{k}}\left|\psi_{m \mathbf{k}}\right\rangle\left\langle\psi_{m \mathbf{k}}\right|=\hat{1}$ into Eq. (10),

$$
\Delta \varepsilon_{n \mathbf{k}}^{\mathrm{DW}}(T)=\sum_{m} \Delta \varepsilon_{n \mathbf{k}, m}^{\mathrm{ORDW}}(T)
$$

where

$$
\begin{aligned}
\Delta \varepsilon_{n \mathbf{k}, m}^{\mathrm{ORDW}}(T) & \\
= & \operatorname{Re} \frac{i}{N_{p}} \sum_{\mathbf{q}, v} \sum_{\kappa, \alpha, \alpha^{\prime}} l_{\mathbf{q} \nu}^{2} \frac{M_{p}}{M_{\kappa}} e_{\kappa \alpha, v}^{*}(\mathbf{q}) e_{\kappa \alpha^{\prime}, v}(\mathbf{q}) \\
& \times\left[h_{m n, \kappa \alpha}(\mathbf{k}, \mathbf{0})\right]^{*}\left\langle u_{m \mathbf{k}}\left|\hat{p}_{\alpha^{\prime}}\right| u_{n \mathbf{k}}\right\rangle_{\mathrm{uc}}\left[2 n_{\mathbf{q} v}(T)+1\right] .
\end{aligned}
$$

Further, by using the relation derived by Lihm and Park [21]

$$
\sum_{\kappa} h_{m n, \kappa \alpha}(\mathbf{k}, \mathbf{0})=i\left(\varepsilon_{m \mathbf{k}}-\varepsilon_{n \mathbf{k}}\right)\left\langle u_{m \mathbf{k}}\left|\hat{p}_{\alpha}\right| u_{n \mathbf{k}}\right\rangle_{\mathrm{uc}}
$$

Eq. (12) is rewritten as

$$
\begin{aligned}
\Delta \varepsilon_{n \mathbf{k}, m}^{\mathrm{ORDW}}(T)= & -\operatorname{Re} \frac{1}{N_{p}} \sum_{\mathbf{q}, v} \sum_{\kappa, \alpha, \kappa^{\prime}, \alpha^{\prime}} l_{\mathbf{q} v}^{2} \frac{M_{p}}{M_{\kappa}} e_{\kappa \alpha, v}^{*}(\mathbf{q}) e_{\kappa \alpha^{\prime}, v}(\mathbf{q}) \\
& \times \frac{\left[h_{m n, \kappa \alpha}(\mathbf{k}, \mathbf{0})\right]^{*} h_{m n, \kappa^{\prime} \alpha^{\prime}}(\mathbf{k}, \mathbf{0})}{\varepsilon_{n \mathbf{k}}-\varepsilon_{m \mathbf{k}}+i \eta}\left[2 n_{\mathbf{q} v}(T)+1\right] .
\end{aligned}
$$

Here the convergence factor is inserted. The momentum operator in Eq. (12) simplifies relating the ORDW term with the symmetry of the material and, thus, with the OSR inherent in the system. Further, owing to the momentum operator, the ORDW term $\Delta \varepsilon_{n \mathbf{k}, m}^{\mathrm{ORDW}}(T)$ vanishes for a pair of KS orbitals that are optically forbidden. The optically allowed combination is expressed as [25]

$$
\left[\Gamma\left(u_{m \mathbf{k}}\right) \otimes \Gamma\left(u_{n \mathbf{k}}\right)\right] \cap \Gamma(x, y, z) \neq \emptyset,
$$

where $\otimes$ is the operator for the direct product, and $\Gamma\left(u_{n \mathbf{k}}\right)$ and $\Gamma(x, y, z)$ are the irreducible representations belonging to the $\left|u_{n \mathbf{k}}\right\rangle$ and Cartesian coordinates, respectively. The OSR is listed in Table I for crystals that have point group $O_{h}$ or $T_{d}$, which include diamond-type and zinc-blende-type materials, respectively.

\section{Computational details}

We calculated the ORDW terms for several diamond-type and zinc-blende-type materials: $\mathrm{C}, \mathrm{Si}, \mathrm{Ge}, \mathrm{SiC}, \mathrm{BN}$, and $\mathrm{BP}$. The calculation was performed based on density functional theory (DFT) $[26,27]$ within the semilocal approximations for the exchange-correlation functional. The generalized gradient approximation (GGA) developed by Perdew, Burke, and Ernzerhof [28] was used except for Ge, for which the local density approximation (LDA) was used to avoid the bandgap vanishing problem. It should also be noted that the zero-point

\begin{tabular}{|c|c|c|c|c|c|c|c|c|c|c|}
\hline$O_{h}$ & $A_{1 g}$ & $A_{2 g}$ & $E_{g}$ & $T_{1 g}$ & $T_{2 g}$ & $A_{1 u}$ & $A_{2 u}$ & $E_{u}$ & $T_{1 u}$ & $T_{2 u}$ \\
\hline $\begin{array}{l}A_{1 g} \\
A_{2 g}\end{array}$ & & & & & & & & & + & + \\
\hline$E_{g}$ & & & & & & & & & + & $\begin{array}{l}+ \\
+\end{array}$ \\
\hline$T_{1 g}$ & & & & & & + & & + & + & + \\
\hline$T_{2 g}$ & & & & & & & + & + & + & + \\
\hline$A_{1 u}$ & & & & + & & & & & & \\
\hline$A_{2 u}$ & & & & & + & & & & & \\
\hline$E_{u}$ & & & & + & + & & & & & \\
\hline$T_{1 u}$ & + & & + & + & + & & & & & \\
\hline$T_{2 u}$ & & + & + & + & + & & & & & \\
\hline$T_{d}$ & $A_{1}$ & $A_{2}$ & & $E$ & $T_{1}$ & $T_{2}$ & & & & \\
\hline$A_{1}$ & & & & & & + & & & & \\
\hline$A_{2}$ & & & & & + & & & & & \\
\hline$E$ & & & & & + & + & & & & \\
\hline$T_{1}$ & & + & & + & + & + & & & & \\
\hline$T_{2}$ & + & & & + & + & + & & & & \\
\hline
\end{tabular}
lattice expansion effect is also important for Ge [29], but we did not consider it in this paper. We used the optimized norm conserving Vanderbilt (ONCV) pseudopotentials [30] developed by Schlipf and Gygi (SG15) [31] and the plane
TABLE I. OSR of diamond-type materials (point group $O_{h}$; upper) and zinc-blende-type materials (point group $T_{d}$; lower). + denotes an allowed transition.

wave cutoff of 60 Ry for wave functions and 240 Ry for charge densities. Furthermore, the electronic and phonon structure was obtained using the QUANTUM ESPRESSO package [22,23].

The primitive cell was optimized theoretically to obtain the lattice constants $6.717,10.347,9.522,8.261,6.805$, and 8.581 $a_{0}$ for $\mathrm{C}, \mathrm{Si}, \mathrm{Ge}, \mathrm{SiC}, \mathrm{BN}$, and $\mathrm{BP}$, respectively, where $a_{0}$ is the Bohr radius. For the convergence factor $\eta$ of the FM and DW terms, we adopted a value of $0.1 \mathrm{eV}$ following our convergence study [32]. We used the Monkhorst-Pack grids of size $8 \times 8 \times 8$ for the electronic and phonon structure calculations, while for the calculation of the FM and DW terms, we used a fine grid of size $20 \times 20 \times 20$ with Fourier interpolation. We used 15 as the number of bands to sum over in the FM [Eq. (7)] and DW [Eq. (11)] terms. See Supplemental Material [32], which includes Refs. [21-23,33], for details on the convergence study of the grids, bands, and convergence factor $\eta$.

\section{RESULTS AND DISCUSSIONS}

\section{A. The DW terms for diamond-type and zinc-blende-type materials}

We now detail the meaning of the DW term specifically for diamond-type and zinc-blende-type materials. For these materials,

$$
\begin{aligned}
& \sum_{\mathbf{q}, v} l_{\mathbf{q} \nu}^{2} e_{\kappa \alpha, v}^{*}(\mathbf{q}) e_{\kappa \alpha^{\prime}, v}(\mathbf{q})\left[2 n_{\mathbf{q} v}(T)+1\right] \\
& \quad=\delta_{\alpha, \alpha^{\prime}} \sum_{\mathbf{q}, v} l_{\mathbf{q} \nu}^{2}\left|e_{\kappa 1, v}(\mathbf{q})\right|^{2}\left[2 n_{\mathbf{q} v}(T)+1\right]
\end{aligned}
$$

holds because of the point group symmetry. Then, the ORDW term [Eq.(12)] can be rewritten as

$$
\begin{aligned}
& \Delta \varepsilon_{n \mathbf{k}, m}^{\mathrm{ORDW}}(T) \\
& \quad=\operatorname{Re} i \sum_{\kappa, \alpha}\left\langle\Delta \hat{\tau}_{\kappa}^{2}\right\rangle_{T}\left[h_{m n, \kappa \alpha}(\mathbf{k}, \mathbf{0})\right]^{*}\left\langle u_{m \mathbf{k}}\left|\hat{p}_{\alpha}\right| u_{n \mathbf{k}}\right\rangle_{\mathrm{uc}},
\end{aligned}
$$




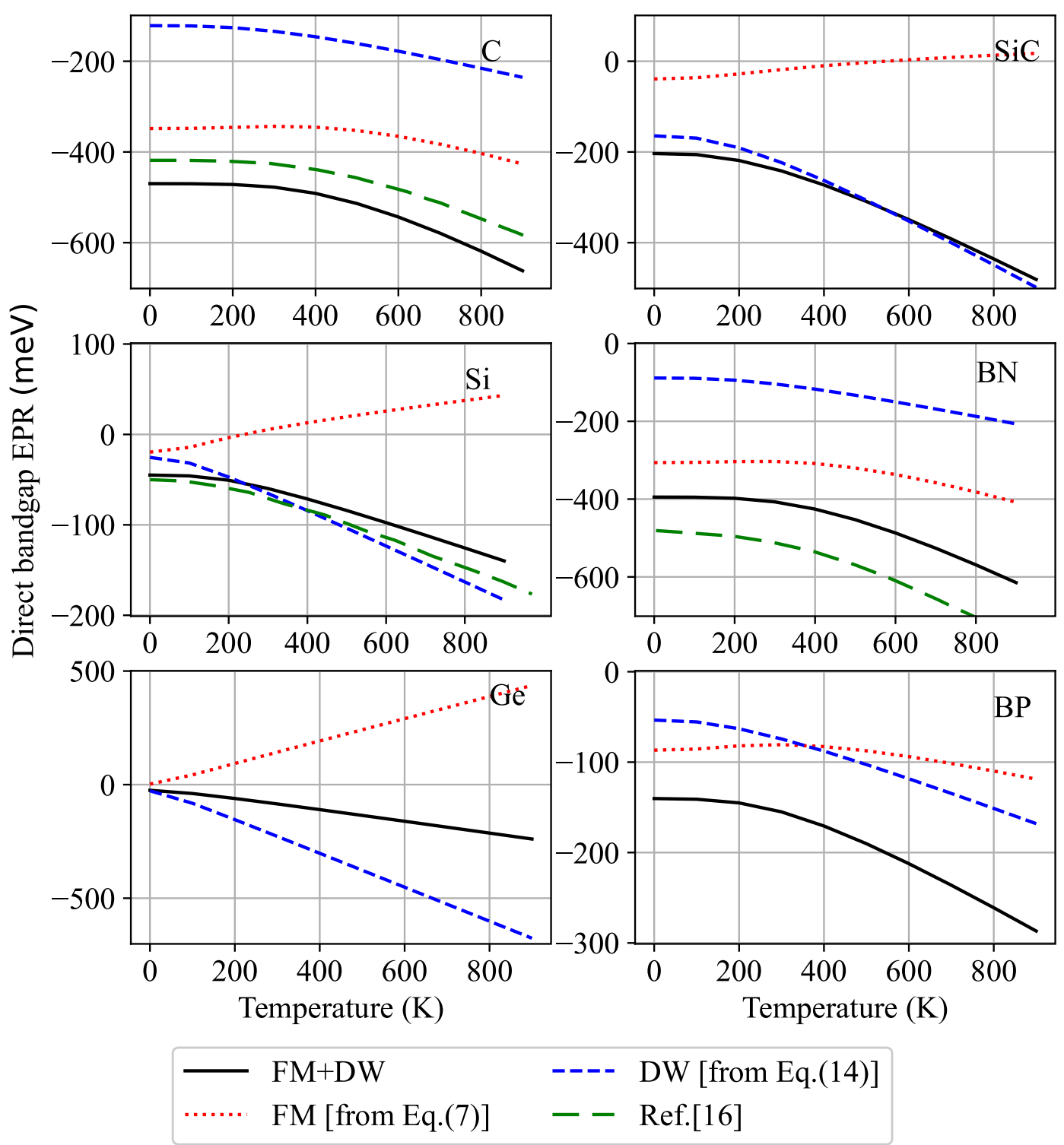

FIG. 1. The direct bandgap EPR (black solid line) together with the FM (red dotted line) and DW (blue dashed line) terms. The direct bandgap EPR from a previous study [16] is also shown (green long-dashed line).

where the MSD of a nucleus $\kappa$ at a temperature $T$ is

$$
\left\langle\Delta \hat{\tau}_{\kappa}^{2}\right\rangle_{T}=\frac{M_{p}}{N_{p} M_{\kappa}} \sum_{\mathbf{q}, v} l_{\mathbf{q} v}^{2}\left|e_{\kappa 1, v}(\mathbf{q})\right|^{2}\left[2 n_{\mathbf{q} v}(T)+1\right]
$$

The ORDW term can then be rewritten using Eq. (13) as

$$
\Delta \varepsilon_{n \mathbf{k}, m}^{\mathrm{ORDW}}(T)=\left(\varepsilon_{m \mathbf{k}}-\varepsilon_{n \mathbf{k}}\right) \frac{\left\langle\Delta \hat{\tau}_{1}^{2}\right\rangle_{T}+\left\langle\Delta \hat{\tau}_{2}^{2}\right\rangle_{T}}{2}
$$

TABLE II. The direct bandgaps at $\mathbf{k}=\mathbf{0}$ and the corresponding EPR at $T=0 \mathrm{~K}$. The first and second columns show our calculated values,

\begin{tabular}{|c|c|c|c|c|}
\hline $\mathrm{C}$ & 5.64 & -470 & $-320[2],-450[2]$ & $-416[29],-415.8[16]$ \\
\hline $\mathrm{Ge}$ & 2.57 & -25 & $-45[35]$ & - \\
\hline $\mathrm{SiC}$ & 6.14 & -203 & - & $-213[36]$ \\
\hline $\mathrm{BN}$ & 8.86 & -395 & - & $-460[36],-502.0[16]$ \\
\hline
\end{tabular}
the third shows experimental data, and the fourth shows theoretical data with AHC theory. 
TABLE III. The MSDs at $T=0 \mathrm{~K}\left(10^{-3} a_{0}^{2}\right)$. The first and second columns show our calculated values and the third shows the experimental data at $1 \mathrm{~K}$. The difference of $1 \mathrm{~K}$ is insignificant in this case.

\begin{tabular}{lccc}
\hline \hline & $\left\langle\Delta \hat{\tau}_{1}^{2}\right\rangle_{0 \mathrm{~K}}$ & $\left\langle\Delta \hat{\tau}_{2}^{2}\right\rangle_{0 \mathrm{~K}}$ & $\left\langle\Delta \hat{\tau}^{2}\right\rangle_{1 \mathrm{~K}}($ Exp.) [37] \\
\hline $\mathrm{C}$ & 5.723 & 5.723 & 5.816 \\
$\mathrm{Si}$ & 8.727 & 8.727 & 8.661 \\
$\mathrm{Ge}$ & 5.319 & 5.319 & 6.065 \\
$\mathrm{SiC}$ & 7.976 & 5.518 & - \\
$\mathrm{BN}$ & 6.997 & 5.689 & - \\
$\mathrm{BP}$ & 9.521 & 5.656 & - \\
\hline
\end{tabular}
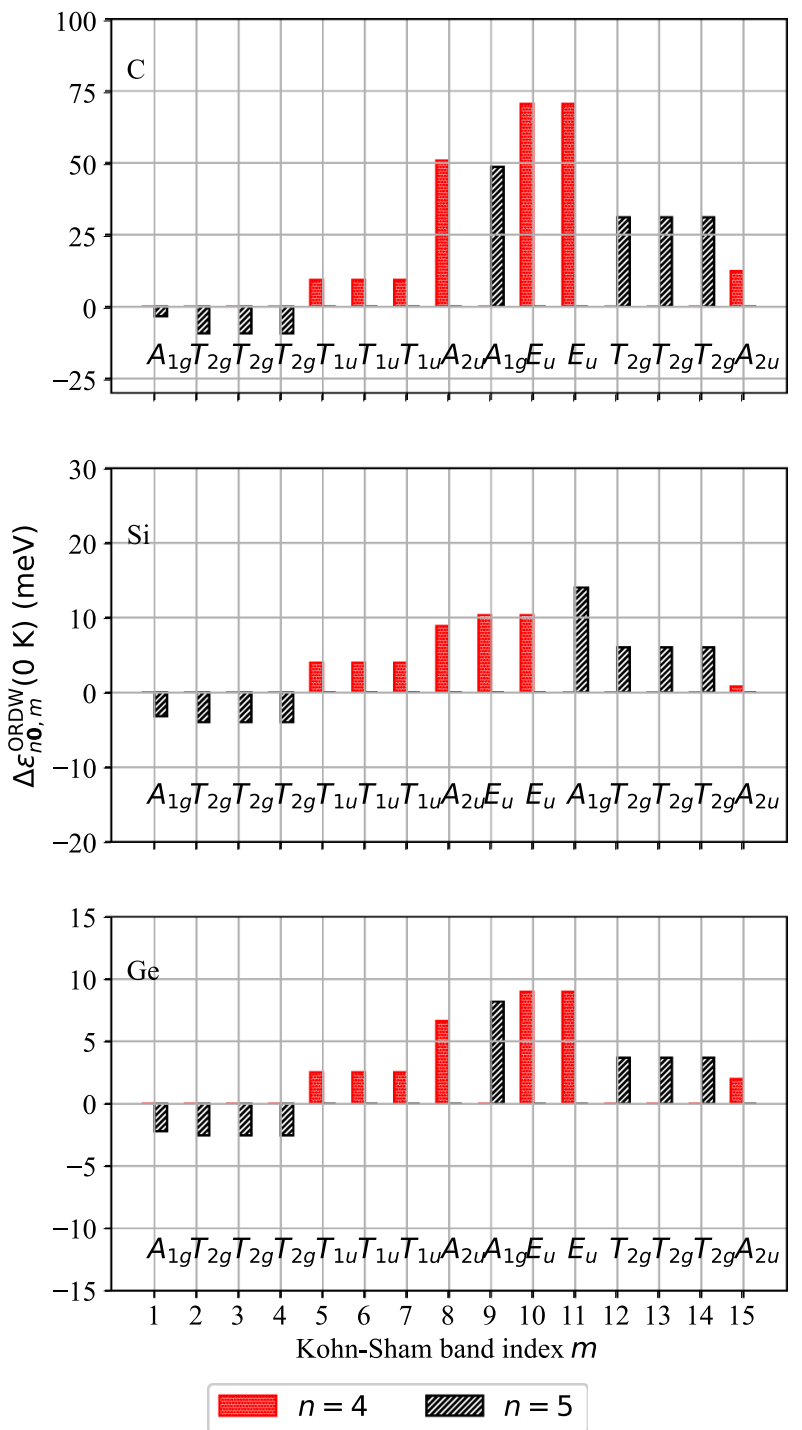

FIG. 2. The ORDW terms, $\Delta \varepsilon_{n \mathbf{n}, m}^{\mathrm{ORDW}}(0 \mathrm{~K})$, for $\mathrm{C}, \mathrm{Si}$, and $\mathrm{Ge}$. Plots are given for the valence top, $n=4$ (shaded with red), and the conduction bottom, $n=5$ (hatched with black). The horizontal axis is the KS band index $m$ whose irreducible representation is also shown. Note that the successive states with the same irreducible representation, $E$ and $T$, are doubly and triply degenerate, respectively, and the value of $\Delta \varepsilon_{n \mathbf{0}, m}^{\mathrm{ORDW}}(0 \mathrm{~K})$ has been averaged over the degenerate states.

$$
\begin{aligned}
& \times \sum_{\alpha}\left|\left\langle u_{n \mathbf{k}}\left|\hat{p}_{\alpha}\right| u_{m \mathbf{k}}\right\rangle_{\mathrm{uc}}\right|^{2} \\
& +\operatorname{Re} i \frac{\left\langle\Delta \hat{\tau}_{1}^{2}\right\rangle_{T}-\left\langle\Delta \hat{\tau}_{2}^{2}\right\rangle_{T}}{2} \\
& \times \sum_{\alpha}\left[h_{m n, 1 \alpha}(\mathbf{k}, \mathbf{0})-h_{m n, 2 \alpha}(\mathbf{k}, \mathbf{0})\right]^{*} \\
& \times\left\langle u_{m \mathbf{k}}\left|\hat{p}_{\alpha}\right| u_{n \mathbf{k}}\right\rangle_{\mathrm{uc}} .
\end{aligned}
$$

For diamond-type materials, where $\left\langle\Delta \hat{\tau}_{1}^{2}\right\rangle_{T}=\left\langle\Delta \hat{\tau}_{2}^{2}\right\rangle_{T}$ holds, the MSD can be represented by the average of these values denoted as $\left\langle\Delta \hat{\tau}^{2}\right\rangle_{T}$. Subsequently, the second term on the right-hand side of Eq. (19) vanishes. Therefore, we obtain

$$
\Delta \varepsilon_{n \mathbf{k}, m}^{\mathrm{ORDW}}(T) \simeq\left(\varepsilon_{m \mathbf{k}}-\varepsilon_{n \mathbf{k}}\right)\left\langle\Delta \hat{\tau}^{2}\right\rangle_{T} \sum_{\alpha}\left|\left\langle u_{n \mathbf{k}}\left|\hat{p}_{\alpha}\right| u_{m \mathbf{k}}\right\rangle_{\mathrm{uc}}\right|^{2},
$$
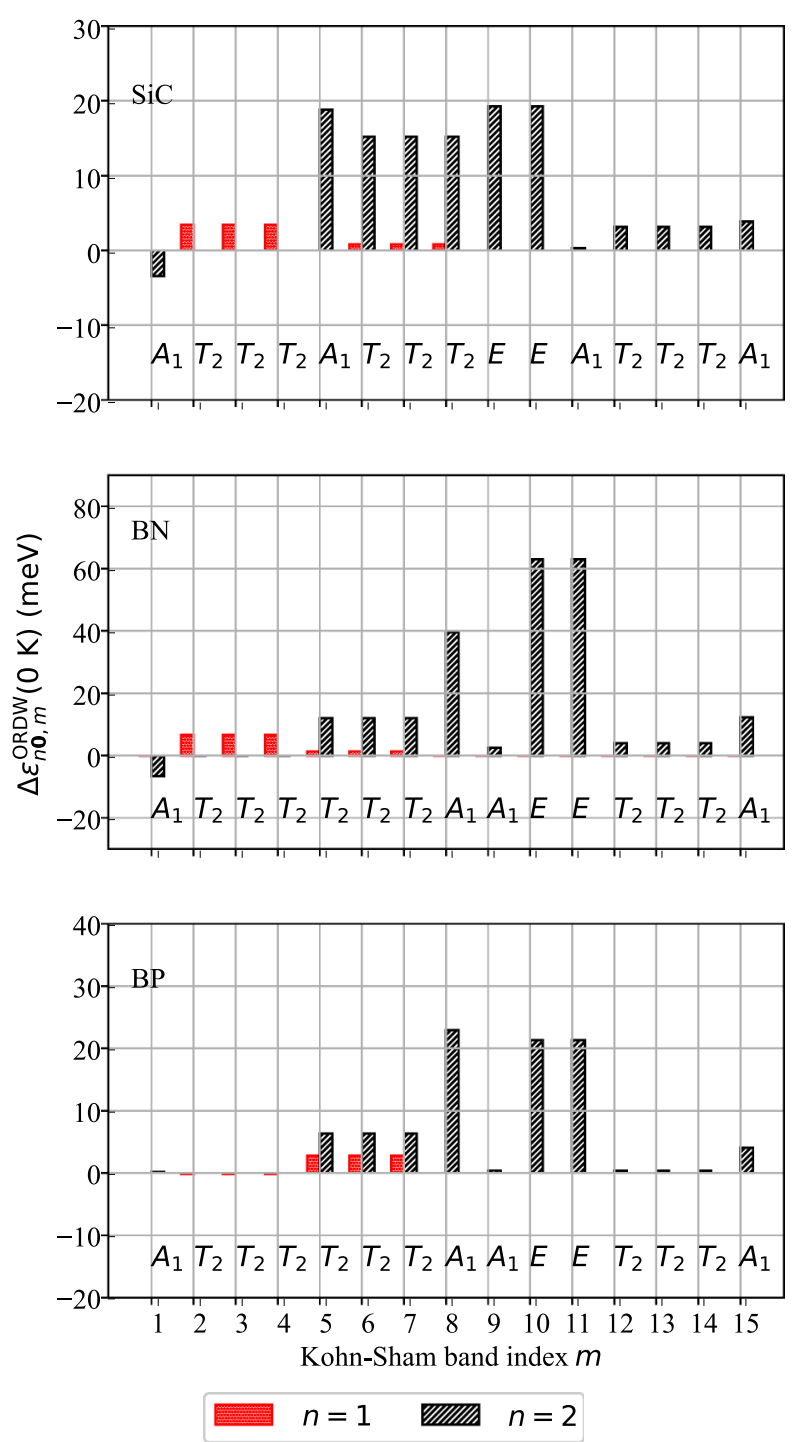

FIG. 3. The ORDW terms, $\Delta \varepsilon_{n \mathbf{0}, m}^{\mathrm{ORDW}}(0 \mathrm{~K})$, for $\mathrm{SiC}, \mathrm{BN}$, and BP. Plots are shown for the lowest valence, $n=1$ (shaded with red) and the valence top, $n=2$ (hatched with black). Details of the notation are the same as in Fig. 2. 

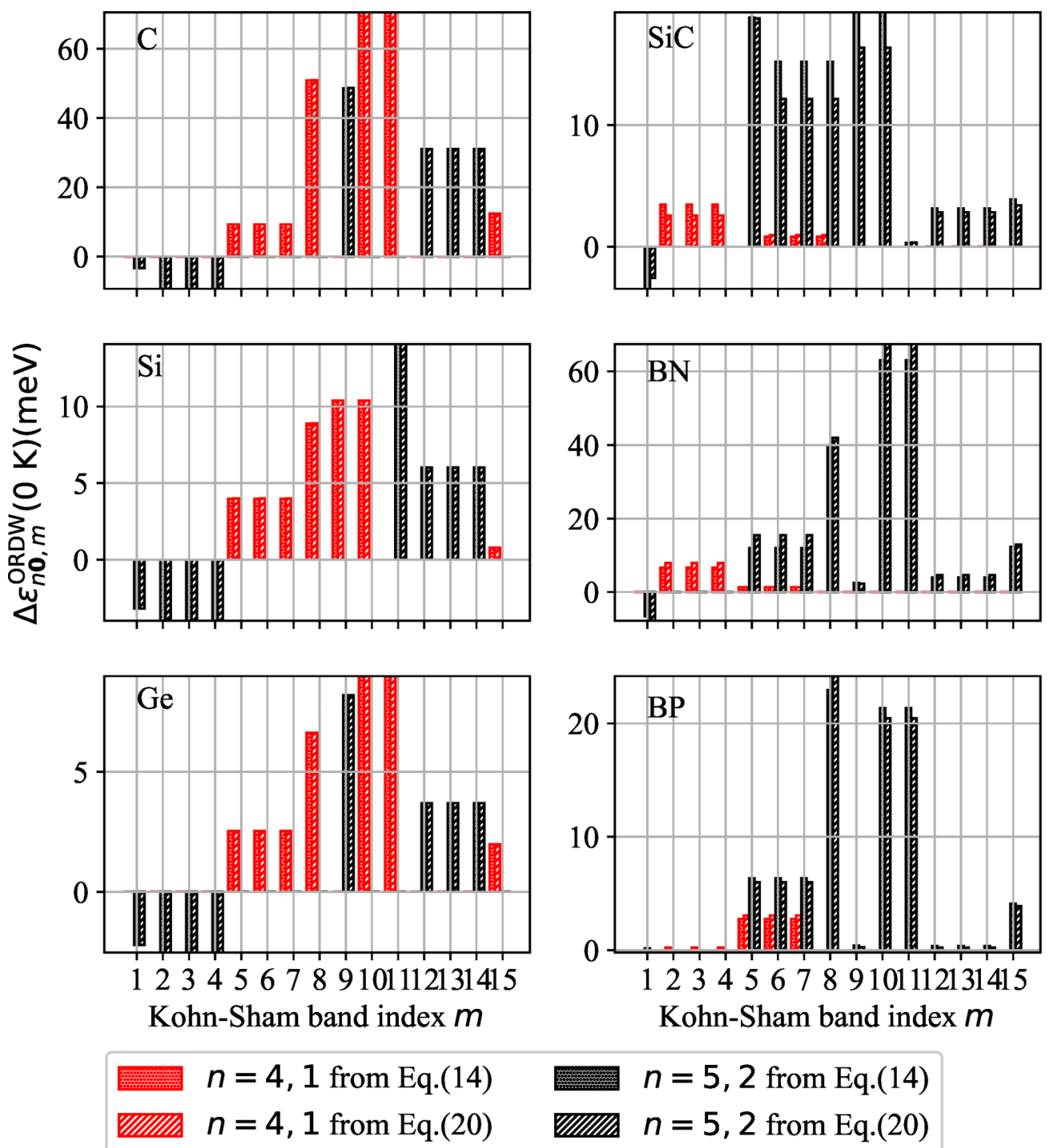

FIG. 4. Comparison of the ORDW terms $\Delta \varepsilon_{n \mathbf{0}, m}^{\mathrm{ORDW}}(0 \mathrm{~K})$ obtained with the original formula [Eq. (14)] with those obtained with the approximate one [Eq. (20)]. Details of the notation are similar to those in Figs. 3 and 4.

which is given as the product of the optical transition energy, MSD, and dipole transition probability, and thus the DW term can be evaluated using these known properties of the materials. For zinc-blende-type materials, Eq. (20) will be valid when the MSD is nearly symmetric $\left\langle\Delta \hat{\tau}_{1}^{2}\right\rangle_{T} \simeq\left\langle\Delta \hat{\tau}_{2}^{2}\right\rangle_{T}$; the validation is presented in subsequent sections.

\section{B. Numerical validation of the direct bandgap EPR and ORDW term}

Before examining the applicability of Eq. (20), we checked our computational setup. We have computed the direct bandgap EPR at $\mathbf{k}=\mathbf{0}$ in the temperature range 0-900 K and the MSD at $0 \mathrm{~K}$. Figure 1 shows that our EPR value is reasonably consistent with the prior calculations done for $\mathrm{C}$, $\mathrm{Si}$, and $\mathrm{BN}$ [16]. The minor difference may have originated from different computational conditions. Table II shows that the direct bandgap EPR at $0 \mathrm{~K}$ is consistent with experimental and theoretical values, although typically differing by 20
$70 \mathrm{meV}$. Table III shows that the calculated MSD at $0 \mathrm{~K}$ differs from the experimental value by only $0.1 \times 10^{-3} a_{0}^{2}$ for $\mathrm{C}$ and $\mathrm{Si}$, while the difference is much larger, $0.7 \times 10^{-3} a_{0}^{2}$, for Ge. The larger difference found for Ge may be due at least partially to the LDA used for the exchange-correlation potential. As mentioned above, we have used LDA to avoid too small a bandgap given by GGA, and we intend to carry out more accurate calculations in a future study. Despite a certain amount of inaccuracy in the present calculation, it is worth emphasizing that the magnitude of EPR is one order of magnitude larger for $\mathrm{C}$ compared with $\mathrm{Si}$ and $\mathrm{Ge}$, and explaining this material dependence is the main objective of our study. As an additional validation of the numerical accuracy, we examined the OSR. Figure 2 shows the plot of the ORDW terms $\Delta \varepsilon_{n \mathbf{0}, m}^{\mathrm{ORDW}}(0 \mathrm{~K})$ of diamond-type materials. As a demonstration, we show the results only for $n=4$ (the valence top) and $n=5$ (the conduction bottom) with $m=$ $1-15$ being considered. We find that the value is zero for all the optically forbidden pairs of $n$ and $m$ (see Table I left for the OSR). 
TABLE IV. Momentum matrix elements $\sum_{\alpha}\left|\left\langle u_{m 0}\left|\hat{p}_{\alpha}\right| u_{n \mathbf{0}}\right\rangle_{\mathrm{uc}}\right|^{2}$ $(n=4,5)$ of diamond-type materials $\left(a_{0}^{-2}\right)$. Values are averaged over degenerate states. Transitions are from the valence top $\left(T_{2 g}\right)$ or conduction bottom $\left(T_{1 u}\right)$ states to the nearest singly $(A)$, doubly $(E)$, or triply $(T)$ degenerate states. Values in parentheses are the transition energies $(\mathrm{eV})$.

\begin{tabular}{lccc}
\hline \hline & $T_{2 g} \rightarrow T_{1 u}$ & $T_{2 g} \rightarrow A_{2 u}$ & $T_{2 g} \rightarrow E_{u}$ \\
\hline $\mathrm{C}$ & $0.096(5.64)$ & $0.655(13.6)$ & $0.230(26.8)$ \\
$\mathrm{Si}$ & $0.060(2.55)$ & $0.333(3.06)$ & $0.077(7.66)$ \\
$\mathrm{Ge}$ & $0.062(2.57)$ & $0.375(3.32)$ & $0.080(10.5)$ \\
& $T_{1 u} \rightarrow A_{1 g}$ & $T_{1 u} \rightarrow T_{2 g}$ & $T_{1 u} \rightarrow A_{1 g}$ \\
\hline $\mathrm{C}$ & $0.021(-27.2)$ & $0.096(-5.64)$ & $0.615(13.8)$ \\
$\mathrm{Si}$ & $0.026(-14.3)$ & $0.060(-2.55)$ & $0.304(5.30)$ \\
$\mathrm{Ge}$ & $0.022(-18.6)$ & $0.062(-2.57)$ & $0.341(4.52)$ \\
\hline \hline
\end{tabular}

A similar validation was performed for zinc-blende-type materials, the results of which are shown in Fig. 3 only for $n=1$ (the lowest valence) and $n=2$ (the valence top). The results indicate that the value of $\Delta \varepsilon_{n \mathbf{0}, m}^{\mathrm{ORDW}}(0 \mathrm{~K})$ is zero for all the optically forbidden pairs without exception (see Table I right for the OSR).

\section{The origin of the material dependence of the ORDW term}

Now we compare the ORDW term obtained using the original formula [Eq. (14)] with that obtained using the approximate one [Eq. (20)], as plotted in Fig. 4. In the diamondtype case, perfect agreement can be found between the rigorous and the approximate formulas. In the zinc-blende case, the match is not perfect, but the difference is within several percent.

It is noteworthy that the ORDW terms are larger for C than for $\mathrm{Si}$ and Ge by an order of magnitude. This striking material dependence primarily originates from the generally larger spacing between the energy bands $\varepsilon_{m \mathbf{k}}-\varepsilon_{n \mathbf{k}}$ and the momentum matrix element $\left|\left\langle u_{m \mathbf{k}}\left|\hat{p}_{\alpha}\right| u_{n \mathbf{k}}\right\rangle_{\text {uc }}\right|$ for $\mathrm{C}$ and not from its MSD $\left\langle\Delta \hat{\tau}^{2}\right\rangle_{T}$, which is different from that of Si and Ge by up to about $50 \%$ (Table III). Table IV shows that the energy and momentum of $\mathrm{C}$ are greater than those of $\mathrm{Si}$ and Ge for almost all transitions.

Although this trend is applicable to diamond-type materials where the material dependence is large, it is not necessarily true when discussing zinc-blende-type materials with minor material dependence (Fig. 4). In addition, the MSD is considerably asymmetric in $\kappa$ for $\mathrm{SiC}$ and $\mathrm{BP}$ (Table III), contrary to the assumption made in deriving Eq. (20). However, generally large values for the ORDW term are found for $\mathrm{BN}$ compared with those of $\mathrm{SiC}$ and $\mathrm{BP}$, and thus this material dependence is correlated to the larger spacing of the energy bands and momentum matrix elements of $\mathrm{BN}$. Hence, the manner in which the approximate ORDW terms may be summed up to recover the DW term is of interest. However, strict investigation is hampered by the slow convergence of Eq. (11). We therefore compared the value obtained by summing the first 15 orbitals using the original formula [Eq.(14)] with that obtained using the approximate formula [Eq.(20)]. The DW terms of the direct bandgap EPR

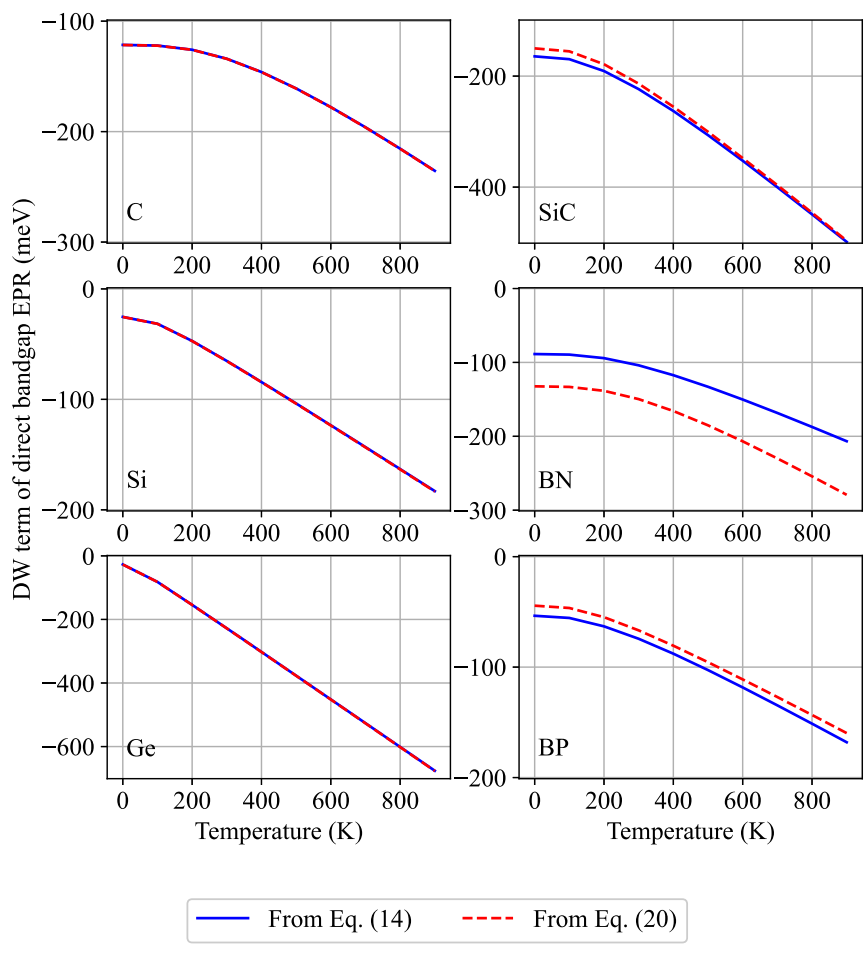

FIG. 5. The DW terms of the direct bandgap EPR $\Delta \varepsilon_{50}^{\mathrm{DW}}(T)-$ $\Delta \varepsilon_{40}^{\mathrm{DW}}(T)$ obtained by summing the first 15 bands, or $m=1$ to 15 , using the original formula [Eq. (14)] and those obtained using the approximate formula [Eq. (20)]. The original is plotted with blue solid line while the approximate is plotted with a dashed red line.

obtained from these formulas are plotted in Fig. 5. The difference between the two is zero for diamond-type materials but not for zinc-blende-type materials. The difference in behavior between diamond-type and zinc-blende-type materials is due to the approximation $\left\langle\Delta \hat{\tau}_{1}^{2}\right\rangle_{T}=\left\langle\Delta \hat{\tau}_{2}^{2}\right\rangle_{T}$. Finally, we discuss finite temperature. Because Eq. (20) can be rewritten as $\Delta \varepsilon_{n \mathbf{k}, m}^{\text {ORDW }}(T) \simeq\left\langle\Delta \hat{\tau}^{2}\right\rangle_{T} /\left\langle\Delta \hat{\tau}^{2}\right\rangle_{0 \mathrm{~K}} \Delta \varepsilon_{n \mathbf{k}, m}^{\mathrm{ORDW}}(0 \mathrm{~K})$, the temperature dependence of the DW term is highly dependent on the temperature dependence of the MSD. However, the material dependence of $\Delta \varepsilon_{n \mathbf{k}}^{\mathrm{DW}}(0 \mathrm{~K})$ affects it as a multiplier.

\section{CONCLUSION}

We reformulated the DW term of the AHC theory to decompose it into the ORDW terms using the formula recently derived by Lihm and Park [21], thereby relating the DW term with the optical dipole transition. For diamondand zinc-blende-type materials, we found that the ORDW term can be factorized into the difference in the KS eigenvalues, the MSD, and the dipole transition probability, as shown in Eq. (20) under the approximation of symmetric MSD for the zinc-blende-type. Further, the OSR and approximate factorization were numerically examined for $\mathrm{C}, \mathrm{Si}$, $\mathrm{Ge}, \mathrm{SiC}, \mathrm{BN}$, and BP using first-principles DFT calculations and were found to reproduce the nonapproximated results perfectly for diamond-type materials and reasonably well for zinc-blende-type materials. It is therefore possible to estimate the magnitude of the DW term from the known properties of materials before performing elaborate EPI calculations. In 
addition, it is possible to ascribe the larger DW term of $\mathrm{C}$ to the larger separation of the electronic bands and momentum matrix elements using our formula. In this way, the DW term is given a physical meaning and its material dependence is given an intuitive explanation. Although the applicability of our results is limited to crystal structures with high symmetry, our results can be generalized to estimate the DW term for materials with low anisotropy. Furthermore, although we have focused only on the DW term, further examination of the whole theory may be important to deepen our understanding of the bandgap EPR.

\section{ACKNOWLEDGEMENT}

The calculations were performed with the facilities of the Supercomputer Center, the Institute for Solid State Physics, the University of Tokyo.
[1] F. Giustino, Electron-phonon interactions from first principles, Rev. Mod. Phys. 89, 015003 (2017).

[2] S. Logothetidis, J. Petalas, H. M. Polatoglou, and D. Fuchs, Origin and temperature dependence of the first direct gap of diamond, Phys. Rev. B 46, 4483 (1992).

[3] S. Baroni, S. de Gironcoli, A. Dal Corso, and P. Giannozzi, Phonons and related crystal properties from density-functional perturbation theory, Rev. Mod. Phys. 73, 515 (2001).

[4] P. B. Allen and V. Heine, Theory of the temperature dependence of electronic band structures, J. Phys. C 9, 2305 (1976).

[5] P. B. Allen and M. Cardona, Theory of the temperature dependence of the direct gap of germanium, Phys. Rev. B 23, 1495 (1981).

[6] P. B. Allen and M. Cardona, Temperature dependence of the direct gap of Si and Ge, Phys. Rev. B 27, 4760 (1983).

[7] C. E. Patrick and F. Giustino, Quantum nuclear dynamics in the photophysics of diamondoids, Nat. Commun. 4, 2006 (2013).

[8] M. Zacharias, C. E. Patrick, and F. Giustino, Stochastic approach to phonon-assisted pptical absorption, Phys. Rev. Lett. 115, 177401 (2015).

[9] M. Zacharias and F. Giustino, One-shot calculation of temperature-dependent optical spectra and phonon-induced band-gap renormalization, Phys. Rev. B 94, 075125 (2016).

[10] M. Zacharias and F. Giustino, Theory of the special displacement method for electronic structure calculations at finite temperature, Phys. Rev. Res. 2, 013357 (2020).

[11] F. Della Sala, R. Rousseau, A. Görling, and D. Marx, Quantum and thermal fluctuation effects on the photoabsorption spectra of clusters, Phys. Rev. Lett. 92, 183401 (2004).

[12] H. Y. Fan, Temperature dependence of the energy gap in semiconductors, Phys. Rev. 82, 900 (1951).

[13] E. Antončík, On the theory of temperature shift of the absorption curve in non-polar crystals, Czech. J. Phys. 5, 449 (1955).

[14] F. Giustino, S. G. Louie, and M. L. Cohen, Electron-phonon renormalization of the direct band gap of diamond, Phys. Rev. Lett. 105, 265501 (2010).

[15] H. Kawai, K. Yamashita, E. Cannuccia, and A. Marini, Electron-electron and electron-phonon correlation effects on the finite-temperature electronic and optical properties of zincblende GaN, Phys. Rev. B 89, 085202 (2014).

[16] S. Poncé, Y. Gillet, J. Laflamme Janssen, A. Marini, M. Verstraete, and X. Gonze, Temperature dependence of the electronic structure of semiconductors and insulators, J. Chem. Phys. 143, 102813 (2015).

[17] M. Friedrich, A. Riefer, S. Sanna, W. G. Schmidt, and A. Schindlmayr, Phonon dispersion and zero-point renormalization of $\mathrm{LiNbO}_{3}$ from density-functional perturbation theory, J.Phys.: Condens. Matter 27, 385402 (2015).
[18] G. Antonius, S. Poncé, E. Lantagne-Hurtubise, G. Auclair, X Gonze, and M. Côté, Dynamical and anharmonic effects on the electron-phonon coupling and the zero-point renormalization of the electronic structure, Phys. Rev. B 92, 085137 (2015).

[19] C. E. P. Villegas, A. R. Rocha, and A. Marini, Anomalous temperature dependence of the band gap in black phosphorus, Nano Lett. 16, 5095 (2016).

[20] M. Engel, M. Marsman, C. Franchini, and G. Kresse, Electron-phonon interactions using the projector augmentedwave method and Wannier functions, Phys. Rev. B 101, 184302 (2020).

[21] J.-M. Lihm and C.-H. Park, Phonon-induced renormalization of electron wave functions, Phys. Rev. B 101, 121102(R) (2020).

[22] P. Giannozzi et al., QUANTUM ESPRESSO: A modular and opensource software project for quantum simulations of materials, J.Phys.: Condens. Matter 21, 395502 (2009).

[23] P. Giannozzi et al., Advanced capabilities for materials modelling with QUANTUM ESPRESSO, J.Phys.: Condens. Matter 29, 465901 (2017).

[24] S. Poncé, G. Antonius, Y. Gillet, P. Boulanger, J. Laflamme Janssen, A. Marini, M. Côté, and X. Gonze, Temperature dependence of electronic eigenenergies in the adiabatic harmonic approximation, Phys. Rev. B 90, 214304 (2014).

[25] Y. E. Kitaev, A. G. Panfilov, P. Tronc, and R. A. Evarestov, Electron state symmetries and optical transitions in semiconductor superlattices: I. Grown along the [001] direction, J. Phys.: Condens. Matter 9, 257 (1997).

[26] P. Hohenberg and W. Kohn, Inhomogeneous electron gas, Phys Rev. 136, B864 (1964).

[27] W. Kohn and L. J. Sham, Self-consistent equations including exchange and correlation effects, Phys. Rev. 140, A1133 (1965).

[28] J. P. Perdew, K. Burke, and M. Ernzerhof, Generalized Gradient Approximation Made Simple, Phys. Rev. Lett. 77, 3865 (1996).

[29] A. Miglio, V. Brousseau-Couture, E. Godbout, G. Antonius, Y.-H. Chan, S. G. Louie, M. Côté, M. Giantomassi, and X. Gonze, Predominance of non-adiabatic effects in zero-point renormalization of the electronic band gap, npj Comput. Mater. 6, 167 (2020).

[30] D. R. Hamann, Optimized norm-conserving Vanderbilt pseudopotentials, Phys. Rev. B 88, 085117 (2013).

[31] M. Schlipf and F. Gygi, Optimization algorithm for the generation of ONCV pseudopotentials, Comput. Phys. Commun. 196, 36 (2015).

[32] See Supplemental Material at http://link.aps.org/supplemental/ 10.1103/PhysRevB.104.245144 for the additional computa- 
tional information and the convergence studies on the q-grid size, convergence factor, and band cutoff.

[33] X. Gonze, P. Boulanger, and M. Côté, Theoretical approaches to the temperature and zero-point motion effects on the electronic band structure, Ann. Phys. 523, 168 (2011).

[34] P. Lautenschlager, M. Garriga, L. Vina, and M. Cardona, Temperature dependence of the dielectric function and interband critical points in silicon, Phys. Rev. B 36, 4821 (1987).

[35] Y. Yin, D. Yan, F. H. Pollak, M. S. Hybertsen, J. M. Vandenberg, and J. C. Bean, Temperature dependence of the fundamental direct transitions of bulk Ge and two Ge/SiGe multiple-quantum-well structures, Phys. Rev. B 52, 8951 (1995).

[36] H. Shang, J. Zhao, and J. Yang, Assessment of the mass factor for the electron-phonon coupling in solids, J. Phys. Chem. C 125, 6479 (2021).

[37] L.-M. Peng, G. Ren, S. L. Dudarev, and M. J. Whelan, Debye-Waller factors and absorptive scattering factors of elemental crystals, Acta Crystallogr. Sect. A 52, 456 (1996). 